$7-2-2020$

\title{
Innovative Use of mHealth and Clinical Technology for Oncology Clinical Trials in Africa
}

Miriam Mutebi

Rohini Bhatia

Omolola Salako

Fidel Rubagumya

Surbhi Grover

See next page for additional authors

Follow this and additional works at: https://ecommons.aku.edu/eastafrica_fhs_mc_gen_surg

Part of the Surgery Commons 
Authors

Miriam Mutebi, Rohini Bhatia, Omolola Salako, Fidel Rubagumya, Surbhi Grover, and Nazik Hammad 


\title{
Technology for Oncology Clinical Trials in Africa
}

\author{
Miriam Mutebi, MD, MSc${ }^{1}$; Rohini Bhatia, BA ${ }^{2}$; Omolola Salako, MBBS $^{3}$; Fidel Rubagumya, MD4; Surbhi Grover, MD, MPH ; and \\ Nazik Hammad, MD, MEHP6
}

\begin{abstract}
Utilization of clinical technology and mobile health (mHealth) is expanding globally. It is important to reflect on how their usage and application could translate in low- and middle-income country (LMIC) settings. With the exponential growth and advancements of mobile and wireless technologies, LMICs are prime to adapt such technologies to potentially democratize and create solutions to health-related challenges. The role of these technologies in oncology clinical trials continues to expand. The lure of mHealth promises disruptive technology that may change the way clinical trials are designed and conducted in many settings. Its applicability in the African context is currently under consideration. Although potentially of expanding benefit, the role of these technologies requires careful and nuanced evaluation of the context in which they might be applied to harness their full potential, while mitigating possible harms or preventing further deepening of disparities within populations. Moreover, technology and digital innovations are no substitute for poor referral pathways and dysfunctional health systems and can only complement or enhance definite strategies aimed at strengthening these health systems.
\end{abstract}

JCO Global Oncol 6:948-953. ๔ 2020 by American Society of Clinical Oncology

Creative Commons Attribution Non-Commercial No Derivatives 4.0 License (c)(1) ()

\section{INTRODUCTION}

There is an increasing interest and development in the field of mobile health (mHealth) and clinical technology globally. It is important to reflect on how its usage and application could translate in many lowand middle-income country (LMIC) settings. With the exponential growth and advancements of mobile and wireless technologies, LMIC countries are prime to adapt such technologies to democratize and create solutions to a vast range of issues. With an increasing number of human populations using a variety of technology, including mobile phones, laptops, or tablets, companies involved in clinical trials have begun to seek ways to deploy and generate data by leveraging use of these mobile technologies. It is estimated that by 2021 , there will be 1.5 million devices per capita, amounting to a total of 11.6 billion devices, and the average mobile connection speed globally will be $>20$ megabits/s. ${ }^{1}$

The role of these technologies in clinical trials and more specifically in oncology clinical trials continues to expand. The lure of mHealth promises disruptive technology that may change the way clinical trials are conducted in many settings, and its applicability in the African context is under consideration. Although potentially of expanding benefit, the role of these technologies requires a thorough evaluation of the context in which they might be applied and in anticipating and minimizing potential barriers or harms to their use and applicability.

\section{MOBILE PHONE TECHNOLOGY}

Globally, the expansion of use of mHealth technologies has been based on a primary parallel expansion of mobile phone users, from $<1$ billion in 2000 to $>7$ billion in 2015. ${ }^{2}$ As defined by the WHO, mHealth involves the use of short messaging services (SMS); global positioning systems; third-, fourth-, and now fifth-generation mobile telecommunication systems ( $3 G, 4 G$, and $5 G$ ); as well as general packet radio service, smart phone applications, and Bluetooth technology. Which technology is used is dependent on the geographic area and access to network in that area. Within mHealth, there have been various proposed categories of use, some of which were defined by the WHO and are listed in Table $1 .^{3}$

The six types of mHealth technologies identified by the WHO include: SMS text messaging, technologies included PDAs and smartphones, patient monitoring devices, mobile telemedicine/telecare devices, MP3 players, and mobile computing ${ }^{4}$ 
TABLE 1. Use of Mobile Health

Use

\begin{tabular}{l}
\hline Medication compliance \\
\hline Health promotion and disease prevention \\
\hline Awareness raising over health issues \\
\hline Health monitoring and disease surveillance \\
\hline Communication \\
\hline Data collection \\
\hline Mobile telemedicine \\
\hline Point of care and decision support \\
\hline Emergency medical response
\end{tabular}

\section{CURRENT APPLICATIONS OF MHEALTH AND CLINICAL TECHNOLOGY IN HIGH-RESOURCE COUNTRIES}

In 2011, the WHO produced an mHealth report detailing that higher-income countries showed more mHealth activities than lower-income countries, with countries in the European region as the most active and those in the African region as the least active. At the time, although many countries reported $\mathrm{mHealth}$ activity, evaluation of those activities was low (12\%). There was little difference between the number of countries reporting at least one mHealth initiative between the low-income and highincome groups $(77 \% \vee 87 \%){ }^{3}$

Demonstrated benefits of these technologies may vary based on the outcome goal. The WHO has recommended moving from usability as an outcome measure to a health outcomes-based approach, with randomized controlled studies and standardized replicable study designs to assist in standardizing how these technologies are studied. The growth in experimental applications, however, has demonstrated improvements in increased access to knowledge and protected health information (PHI), improved timely access to emergency and general health services, reduced drug shortages at health clinics, and enhanced clinical diagnosis and treatment adherence. ${ }^{3}$

Countries in the high-income group, as denoted by the WHO, were less likely to use mHealth for surveillance initiatives and health surveys, most likely because an existing surveillance capacity already exists in these places. More often, high-income countries (HICs) used mHealth to send appointment reminders to improve patient adherence (eg, missing appointments costs the United Kingdom an estimated 790 million pounds per year). ${ }^{3}$

A more recent review of mHealth application areas in $\mathrm{HICs}$ and LMICs by Abaza and Marschollek described newer apps and technology used. ${ }^{5}$ Of the 210 studies reviewed, 149 were based in HICs versus 61 in LMICs. The majority of health monitoring applications focused on NCDs and were studied in HICs (only 3.6\% in LMICs). In the health monitoring and surveillance technology category, diabetes apps allowed patients to enter glucometer readings and send them to the health care provider for personalized feedback. The outcomes of these evaluation studies focused not only on usability but also on reduced rate of hospitalizations or decrease in duration of hypoglycemic events. Apps for NCDs, addiction, and mental health were more common in HICs. However, the African region did not have any applications for NCD studies, with apps more likely being used for communicable diseases, including HIV and tuberculosis. Even among all the regions studied, NCDs were the least-studied disease group and cancer the least-studied among the 4 NCD categories (diabetes, CVD, respiratory disease, and cancer). ${ }^{5}$

\section{CURRENT APPLICATIONS OF MHEALTH AND CLINICAL TECHNOLOGY IN AFRICA AND OPPORTUNITIES FOR CLINICAL TRIAL USE}

Data from the International Telecommunication Union showed that of the 6 billion mobile phone subscriptions globally in 2011, > 80\% of the 660 million new subscriptions that year were from developing countries. ${ }^{6}$ The upsurge of mobile phone use and penetration has resulted in an increase in availability, more so than infrastructure like electricity, paved roads, or running water., ${ }^{7,8}$

A preliminary systematic review of seven mHealth projects in LMICs suggested a potential improvement in health communication between patients and health workers. ${ }^{9}$ Another systematic review of the use of mHealth in Africa revealed that out of 44 projects conducted in Africa between 2006 and 2013, 19 were pilot studies and 11 were randomized controlled trials. The remainder of the studies were varied and involved mixed-methods, cross-sectional, cohort, and cost analysis studies and qualitative interviews and literature reviews. ${ }^{8}$ Most of the mHealth studies done during this period focused on infectious diseases like HIV, tuberculosis, and malaria.

Among the clinical trials done, a number of studies focused on patient follow-up and adherence to treatment protocols $(n=8)$, staff evaluation, monitoring and compliance with guidelines $(n=2)$, and data collection/transfer and reporting. Although these trials may give a sense of the potential for application in other clinical trials, they were encumbered by the small size of trials and a lack of scalability to see how these strategies could perform in a broader setting. ${ }^{8}$

The role of mHealth in other study designs has been demonstrated, and the utility appears around different functions of patient care in different settings. In Kenya, a middle-income country, mHealth has been used to monitor pharmacies and prevent stock-outs of medication, suggesting a possible use in a clinical trial setting. ${ }^{10}$ A collaborative project in South Africa, an uppermiddle-income country, Project Masilueke sent out 1 million SMS messages per day to subscribers of a local telecommunications operator and project partner to 
encourage HIV/AIDS testing. Although effective in getting the information to subscribers, the impact of these messages on behavior change is less well studied. ${ }^{11}$

mHealth initiatives have also been used to provide staff support and motivation, especially for health workers working in remote or difficult-to-access areas. A pilot project in Botswana showed that it was possible to link specialists and health care workers to improve diagnostic accuracy and patient care. ${ }^{12}$ This project has been replicated in many academic, training, and clinical centers in Africa, where, through use of mobile applications like WhatsApp, Listserv, and so on, health workers are able to link and have discussions around patients. More recently mHealth has demonstrated the ability to link and integrate capacitybuilding strategies around digital health initiatives. ${ }^{13}$ Similar models could potentially be developed and expanded to the clinical trial space.

As relates to oncology and mHealth, a mixed-methods study in Cape Town, South Africa described the feasibility of mobile phones to improve management of patients with cervical cancer precancerous lesions. ${ }^{14}$ In South Africa, mobile phone penetration is $98 \%$, and the use of mHealth, specifically SMS messaging, is increasing. The authors found support among both providers and patients for an SMS colposcopy clinic appointment reminder system as well as the use of mobile phones to convey Papanicolaou test results (or at least, availability of result). A surprising barrier to the success of the study was the high loss of mobile phones (58\% had ever lost a phone, $28 \%$ had lost one in the last year). This also brought up questions of privacy with SMS-based interventions. ${ }^{14}$

Mobile apps have been increasingly used to provide support for cancer survivors, which could be a potential entry point for developing patient-centric clinical trials. Africa still has the highest health worker deficits, with this being particularly more acute in oncology. Through the use of mobile apps, it has now been possible to set up local or regional virtual tumor boards, where patients and their treatment plans are discussed comprehensively. A current initiative by the US National Institutes of Health/ National Cancer Institute, project ECHO, ensures discussion around cancer control strategies and virtual tumor boards for continental participants who can submit and discuss patients with cancer or initiatives with specialists from within and without Africa. ${ }^{15}$ Virtual rounds, such as the radiation oncology chart rounds facilitated by the African Organization for Research and Training in Cancer (AORTIC), help to establish communities of practice and to engage and discuss complex cases where specialists might be few. ${ }^{16}$

All these steps are fundamental to establishing the frameworks of best clinical practice and patient-centered strategies through which clinical trials and other research initiatives can evolve.

\section{CHALLENGES TO THE USE OF TECHNOLOGY IN CLINICAL TRIALS IN AFRICA}

Although mHealth has been in use in several low-income countries (LICs) for different clinical reasons, there are close to no reports of its use in clinical trials. The use of mHealth in clinical trials in HICs has been reported but is projected to possibly have its biggest impact on clinical trials by $2030 .{ }^{17}$ The use of this digital technology has met with several challenges. Participants of a recent survey conducted by KNect365 shared their concerns about the use of mHealth in clinical trials. These involved concerns around data security and privacy, technical operability, acceptability, usability, and internet connections, among others. ${ }^{17}$

\section{Data Security and Privacy}

Clinical trials store participants' data with $\mathrm{PHI}$ that needs protection and privacy. Given the scarcity of internet connection, which itself is a challenge to use of mHealth in LICs, trial participants would be tempted to connect to any wireless internet connection, even those that are unsecured. This may provide the chance for cyber criminals who would use PHI for ill reasons. It would be challenging for the trialists to provide secured internet to all study participants. Researcher who are looking to use mHealth need to make sure that participants' information is protected and access is limited to only authorized users.

A number of strategies have been proposed to counter this, including setting up frameworks for good data governance to ensure protection of vulnerable populations, particularly those in LMICs. These strategies include the development of "ethical oversight and informed consent processes," data protection through the regulation of data access controls, enhancing the sustainability of ethical data use, and developing and enacting legislation relevant to data protection. ${ }^{18}$

\section{System Integration}

Many people in LICs use phones only for calls and text, meaning that most of these phones may not be suitable to be used in clinical trials. Most mHealth health care projects/trials come as mobile applications, especially on smart phones. Smartphone-based research studies can pose an ethical dilemma, as cost of smartphones and the cost of connectivity to smartphones may be prohibitive for a large segment of the population and may lead to selection bias and worsening disparities because of uneven distribution. ${ }^{19}$

Even for smartphones, there are different operating systems; hence, there could be an interoperability challenge among trial participants who have different operating systems. For a trial to be effective, with easy exchange of required information, there would be a need to have the same operating system or a system designed to allow integration with other systems 


\section{Internet Connection}

This includes availability, speed, and strength. As mentioned earlier, rates of internet penetration in LICs are still low. This would be a huge challenge for mHealth projects/ trials that need the use of internet connection for data entry by study participants and auto-backups. There is a need for easy access of internet and also strong and high-speed connections for entry, transmission, and reception of data.

\section{Usability}

Health literacy and numeracy are a concern for many populations in Africa. Individual or general literacy (the ability to read and write) is comparatively lower in Africa and invariably has an effect on health literacy. Despite advances in digital technology, the use of different technologies frequently demands that one have the ability to read and write. Although there is a gradual increase in educational strategies on the continent as part of achieving the sustainable development goals, a significant proportion of populations may face difficulties with the use of technology. In addition, multiple local dialects and languages may further compound patients' abilities to use these devices effectively, as they frequently come in a limited number of languages, the predominant one being English.

Care must be exercised in the use of mobile technology in ethically demanding aspects of clinical trials, such as informed consent. However, the use of technology may mitigate some of these issues and bring new challenges, such as the 2013 Indian Supreme Court ruling, of recording the informed consent process on video. ${ }^{20}$

Usability also refers to the ease of how to learn to use the mobile application by trial participants with different levels of education. If an application is difficult to use, then this might result in poor feedback or inappropriate or wrong data entry. In addition, there is acceptability challenge, which concerns the participants, clinicians, and regulatory bodies. Another challenge is ensuring accuracy of data collection, without which a trial result may not be reliable.

\section{Research Rigor}

Despite the use of mHealth in a number of scenarios, there is a paucity of data on its potential for scalability. Perhaps because of its relative novelty, there are a number of pilot studies that may have shown certain benefits in key areas. These have sometimes not translated into similar findings in randomized trials. The randomized trials have, however, been few, involving small numbers, which may not necessarily be generalizable to large populations. As the oncology research community in Africa incorporates emerging research paradigms, such as pragmatic clinical trials and learning health care systems, ${ }^{21}$ mobile technology may deliver more opportunities and perhaps a different set of challenges than those encountered in traditional clinical trials. More studies and data are required to determine the broader and longer-term effects of the use of digital technology in clinical trials and other research studies.

\section{KEYS TO SUCCESSFUL MHEALTH USE}

Attempts to define what constitutes a successful mHealth project in Africa have been made. Aranda et $a l^{8}$ have crystallized these down to 4 fundamental principles: the availability of a good design of the tool, engagement of stakeholders, the available technology and resources to support the initiative, and integration of the digital health initiatives into the health care systems.

\section{Project Design}

Even as one thinks of the design, one needs to think through the devices or tools one intends to use and to contextualize the tools that one intends to use to one's setting. This comes down to acceptability and use of the devices. Before introducing a digital tool, one needs to be aware of the characteristics of populations, including health literacy and numeracy. Irrespective of design, digital technologies must take into account the unique sociocultural consideration of the population they intend to be used in. For instance, practices such as the use of a single phone per household or the phone being under the care of the household leader could easily hamper the utility of these devices. Converting applications into local languages could potentially increase acceptability and usage of these devices in local settings.

\section{Engagement of Stakeholders}

It is important to involve all parties involved in the potential use and maintenance of these devices early. Developing strong private-public partnerships and robust multidisciplinary teams has been demonstrated to have good outcomes in execution of mHealth projects. ${ }^{8}$

\section{Availability of Technology and Support}

To ensure longevity and sustainability of digital technology, systems need to be established to regularly support and upgrade the existing technology. Building into mHealth components for capacity building that will train local staff to maintain and support these technologies can only serve to enhance and retain successful programs.

\section{Integration Into Existing Health Systems}

A critical part of the any digital technology is that it must successfully be integrated into preexisting health systems rather than as a standalone device. Most governments may have a preexisting digital strategy. Getting appropriate buyin from the partners and designing tools that integrate well and complement existing resources ensures the relevance of the project and its potential for longevity. Integration of these various technologies will enable data mining and analytics from multiple sources. Researchers in Africa must harness the expanding uptake of mobile technology to ensure meaningful contribution of this technology to the dawn of "big data" in Africa and that it will be accessible, 
safe, relevant to the population, and beneficial to health outcomes.

\section{CONSOLIDATION OF MHEALTH SERVICES: TOWARD GREATER REPRODUCIBILITY AND GENERALIZABILITY}

As mHealth becomes more ubiquitous, there have been global attempts to streamline and integrate these strategies into mainstream clinical practice. The WHO has been key in helping to consolidate these efforts. The WHO has also been key in developing resources such as handbooks for the scaling up of digital health initiatives like the monitoring and evaluation of digital health projects: mHealth Assessment and Planning for Scale (MAPS) Toolkit ${ }^{22}$ and the handbook for monitoring and evaluation of digital health. ${ }^{23}$ Although some of these initiatives were initially aimed at primarily improving maternal, child, and adolescent health, they can be expanded to cancer care and other medical areas. In 2018, there was a World Health Assembly resolution calling for a global strategy on digital health to support the drive toward universal health coverage in different countries. ${ }^{24}$ Earlier in 2019, the WHO released the first guidelines with strategies to help strengthen health systems: "WHO Guideline: Recommendations on Digital Interventions for Health System Strengthening."25 These guidelines provide 10 key recommendations on the potential utility of digital health and how it can be harnessed and used within health systems as countries strive toward universal health coverage.

Perhaps of greatest significance is the attempt to streamline the reporting of mHealth projects through the mERAS model, which was developed by a team at Johns Hopkins University in 2016. They developed a 16-item checklist for all mHealth evidence to be reported in keeping with other trials, such as PRISMA for systematic reviews and CONSORT models for clinical trials. This 16 -item checklist looks at the feasibility, usability, and sustainability of different

\author{
AFFILIATIONS \\ ${ }^{1}$ Aga Khan University, Nairobi, Kenya \\ ${ }^{2}$ Sinai Hospital of Baltimore, Baltimore, MD \\ ${ }^{3}$ Lagos University Teaching Hospital, Lagos, Nigeria \\ ${ }^{4}$ Rwanda Military Hospital, University of Global Health Equity, Kigali, \\ Rwanda \\ ${ }^{5}$ University of Pennsylvania, Philadelphia, PA \\ ${ }^{6}$ Queen's University Cancer Center of Southeastern Ontario, Kingston \\ Health Science Center, Kingston, Ontario, Canada
}

\section{CORRESPONDING AUTHOR}

Miriam Mutebi, MD, MSc, Aga Khan University Hospital, 3Rd Parklands Ave, 30270-00100, Nairobi, Kenya; Twitter: @m_mutebi; e-mail: mcmutebi@yahoo.com.

\section{AUTHOR CONTRIBUTIONS}

Conception and design: Miriam Mutebi, Fidel Rubagumya, Surbhi Grover, Nazik Hammad

Collection and assembly of data: Miriam Mutebi, Rohini Bhatia, Omolola Salako, Fidel Rubagumya interventions and technologies. ${ }^{26}$ These steps will ensure that data collected across specialties and interventions is more homogenous, reproducible, and generalizable across populations. This checklist also helps to provide a framework for mHealth interventions in cancer care in different resource settings.

As the choices of smartphone applications and mHealth initiatives continue to expand, what becomes important is the evaluation and added value or cost effectiveness of these applications. Going forward, enhancing legislation to strengthen privacy and security for eHealth EMRS, for example, is a crucial step. In addition, a method to standardize and increase awareness of the already available mHealth options does not yet exist, which is one barrier preventing widespread uptake of these applications. One of the first steps to this might be the uptake and use of the digital health maps coordinated by WHO, where individuals can list their mHealth initiatives, ${ }^{27}$ and the use of the newly launched WHO guidelines and the mERAS checklist. The oncology research community in Africa must collaborate to rethink innovative designs of clinical trials and explore ways to incorporate mHealth and digital technology safely and equitably for purposes of clinical trial expansion in the continent.

In addition, although mHealth and clinical technology may be poised to potentially modify the research landscape, they cannot form a broad panacea for what ails dysfunctional systems. Even the best of technologies cannot substitute for poor referral pathways, disempowered populations, or a lack of access to critical health resources. Key emphasis must be laid on continually strengthening health systems, with digital tools being used to complement already-existing tenets. Even as we embrace the range of new possibilities, the use of virtual tools must always remain firmly grounded in the principles of universal health access.
Data analysis and interpretation: Miriam Mutebi, Fidel Rubagumya Manuscript writing: All authors

Final approval of manuscript: All authors

Accountable for all aspects of the work: All authors

\section{AUTHORS' DISCLOSURES OF POTENTIAL CONFLICTS OF INTEREST}

The following represents disclosure information provided by authors of this manuscript. All relationships are considered compensated unless otherwise noted. Relationships are self-held unless noted. I = Immediate Family Member, Inst = My Institution. Relationships may not relate to the subject matter of this manuscript. For more information about ASCO's conflict of interest policy, please refer to www.asco.org/rwc or ascopubs. org/go/site/misc/authors.html.

Open Payments is a public database containing information reported by companies about payments made to US-licensed physicians (Open Payments).

No potential conflicts of interest were reported. 


\section{REFERENCES}

1. Kakkar AK, Sarma P, Medhi B: mHealth technologies in clinical trial: Opportunities and challenges. Indian J Pharmacol 50:105-107, 2018

2. International Telecommunication Union: ICT Facts \& Figures: The World in 2015. 2015

3. World Health Organization: mHealth: New horizons for health through mobile technologies. WHO: Global Observatory for eHealth Series, Volume 3. World Health Organization, 2011

4. Mechael P, Sloninsky D: Towards the development of an mHealth strategy: A literature review. Geneva, World Health Organization, 2008. http://www.who.int/ goe/mobile_health/mHealth/Review_Aug09.pdf

5. Abaza H, Marschollek M: mHealth application areas and technology combinations. A comparison of literature from high and low/middle income countries. Methods Inf Med 56:e105-e122, 2017

6. International Telecommunication Union: Key statistical highlights: ITU data release June 2012. Geneva, International Telecommunication Union, 2012

7. World Economic Forum, mHealth Alliance: Amplifying the impact: Examining the intersection of mobile health and mobile finance: A discussion guide for collaborative insight presented by the World Economic Forum, in partnership with the mHealth Alliance. Geneva, World Economic Forum, 2011

8. Aranda-Jan CB, Mohutsiwa-Dibe N, Loukanova S: Systematic review on what works, what does not work and why of implementation of mobile health (mHealth) projects in Africa. BMC Public Health 14:188, 2014

9. Hurt K, Walker RJ, Campbell JA, et al: mHealth interventions in low and middle-income countries: A systematic review. Glob J Health Sci 8:54429, 2016

10. Githinji S, Kigen S, Memusi D, et al: Reducing stock-outs of life saving malaria commodities using mobile phone text-messaging: SMS for life study in Kenya. PLoS One 8:e54066, 2013

11. Vital Wave Consulting: mHealth for development: The opportunity of mobile technology for healthcare in the developing world. Washington DC, UN FoundationVodafone Foundation Partnership, 2009 http://www.vitalwaveconsulting.com/pdf/mHealth.pdf

12. Chang AY, Ghose S, Littman-Quinn R, et al: Use of mobile learning by resident physicians in Botswana. Telemed J E Health 18:11-13, 2012

13. Hyder AA, Selig H, Ali J, et al: Integrating capacity development during digital health research: A case study from global health. Glob Health Action 12:1559268, 2019

14. Moodley J, Constant D, Botha MH, et al: Exploring the feasibility of using mobile phones to improve the management of clients with cervical cancer precursor lesions. BMC Womens Health 19:2, 2019

15. National Cancer Institute: NCI/CGH Project ECHO program. https://www.cancer.gov/about-nci/organization/cgh/research/project-echo-program

16. AORTIC: AORTIC radiation oncology special interest. http://aorticconference.org/2017/aortic-radiation-oncology-special-interest/index.html

17. https://knect365.com/clinical-trials-innovation/article/e115b236-9f66-447f-a66d ac7196fc4eab/mhealth-clinical-trials-data-report analysis?utm_ campaign=Share+Widget\&utm_medium=Article+Share\&utm_source=url

18. Tiffin N, George A, LeFevre AE: How to use relevant data for maximal benefit with minimal risk: Digital health data governance to protect vulnerable populations in low-income and middle-income countries. BMJ Glob Health 4:e001395, 2019

19. McConnell M, Ashley E: Mobile health research: App-based trials and informed consent. N Engl J Med 376:856-867, 2017

20. Kang G: Video informed consent. N Engl J Med 376:863-864, 2017

21. Ford I, Norrie J: Pragmatic trials. N Engl J Med 375:454-463, 2016

22. World Health Organization: The MAPS toolkit: mHealth assessment and planning for scale. Geneva, World Health Organization, 2015

23. World Health Organization: Monitoring and evaluation of digital health interventions. https://apps.who.int/iris/bitstream/handle/10665/252183/ 9789241511766-eng.pdf;jsessionid=B7457633E61BAB49A91CF9939267525B? sequence=1

24. World Health Assembly: Digital health resolution. http://apps.who.int/gb/ebwha/pdf_files/WHA71/A71_R7-en.pdf?ua=1

25. World Health Organiztion: WHO guideline: Recommendations on digital interventions for health system strengthening http://www.who.int/reproductivehealth/ publications/digital-interventions-health-system-strengthening/en/

26. Aggarwal S, Lefevre A, Lee J: Guidelines for reporting of health interventions using mobile phones: Mobile health (mHealth) evidence reporting and assessment (mERA) checklist. BMJ 17:352:11174, 2016

27. World Health Organization: Digital health atlas. https://digitalhealthatlas.org/en/-/ 